\title{
Skew Generalized Secant Hyperbolic Distributions: Unconditional and Conditional Fit to Asset Returns
}

\author{
Matthias Fischer \\ Department of Statistics and Econometrics, \\ University of Erlangen-Nuremberg
}

\begin{abstract}
A generalization of the hyperbolic secant distribution which allows for both skewness and leptokurtosis was given by Morris (1982). Recently, Vaughan (2002) proposed another flexible generalization of the hyperbolic secant distribution which has a lot of nice properties but is not able to allow for skewness. For this reason, Fischer and Vaughan (2002) additionally introduced a skewness parameter by means of splitting the scale parameter and showed that most of the nice properties are preserved. We briefly review this class of distributions and apply them to financial return data. By means of the Nikkei225 data, it will be shown that this class of distributions, the socalled skew generalized secant hyperbolic distribution, provides an excellent fit in the context of unconditional and conditional return models.

Zusammenfassung: Eine Generalisierung der Secans hyperbolicus Verteilung, die sowohl Schiefe als auch Leptokurtosis erlaubt, ist in Morris (1982) angegeben. Unlängst wurde in Vaughan (2002) eine weitere flexible Verallgemeinerung vorgeschlagen, die zwar eine Reihe angenehmer Eigenschaften aufweist jedoch keine Schiefe erlaubt. Deshalb führten Fischer and Vaughan (2002) durch Aufteilung des Skalenparameters einen zusätzlichen Schiefeparameter ein und zeigten, dass die meisten angenehmen Eigenschaften dabei erhalten bleiben. Wir besprechen nochmals kurz diese Klasse von Verteilungen und wenden sie dann auf Ertragsdaten an. Bezüglich der Nikkei225 Daten wird gezeigt, dass diese Verteilungsklasse, die so genannte schiefe generalisierte Secans hyperbolicus Verteilung, bei bedingten und nicht-bedingten Ertragsmodellen eine ausgezeichnete Anpassung aufweist.
\end{abstract}

Keywords: Hyperbolic Secant Distribution, SGSH Distribution, NEF-GHS Distribution, Skewness, GARCH.

\section{Introduction}

The hyperbolic secant distribution, which was first studied by Baten (1934) and Talacko (1956), seems to be an appropriate candidate as a starting point for financial return models. Firstly, it exhibits more leptokurtosis than the normal and even more than the logistic distribution. Secondly, the cumulative distribution function admits a closed form implying that, for example, risk neutral probabilities of option prices can be calculated fast and accurate. Thirdly, this distribution is reproductive (i.e., the class is preserved under convolution), infinitely divisible with existing moment-generating function and has finite moments. Since 1956, two generalization have been proposed which both incorporate most of these properties, too and, in addition, allow for a more flexible form concerning skewness and leptokurtosis. 
The first generalization was proposed by Morris (1982) in the context of natural exponential families (NEF) with quadratic variance function (i.e., the variance is a quadratic function of the mean). In this class consisting of six members, one distribution, the socalled NEF-GHS distribution, is generated by the hyperbolic secant distribution. The NEF-GHS distribution allows for skewness and arbitrarily high excess kurtosis. Morris (1982) showed that this class is again reproductive, infinitely divisible with existing moment-generating function and existing moments. However, the corresponding cumulative distribution function doesn't admit a closed form.

Recently, Vaughan (2002) proposes a family of symmetric distributions, the so-called generalized secant hyperbolic (GSH) distribution, with kurtosis ranging from 1.8 to infinity. This family includes both the hyperbolic secant and the logistic distribution and closely approximates the Student t-distribution with corresponding kurtosis. In addition, the moment-generating function and all moments exist, and the cumulative distribution is given in closed form. Unfortunately, this family does not allow for skewness. For this purpose, Fischer and Vaughan (2002) introduce a skewness parameter by means of splitting the scale parameter according to Fernández et al. (1995). This method preserves the closed form for the density, the cumulative distribution function and the inverse cumulative distribution function.

It will be shown that this family, termed as skewed generalized secant hyperbolic distribution (SGSH), provides an excellent fit to the Nikkei225 data. This is verified in the context of unconditional and conditional return models. In particular, we compare the results to other popular models for financial return data which have been proposed in the literature in the past: The $\alpha$-stable distributions (see, e.g., Mittnik et al., 1998), the class of generalized hyperbolic distributions (see, e.g., Prause, 1999), the generalized logistic family of McDonald (1991) and a skewed generalized family of t-distributions of Grottke (2001).

\section{Generalizations of Hyperbolic Secant Distributions}

\section{1 (Generalized) Hyperbolic Secant distribution}

A symmetric random variable $X$ is said to follow a hyperbolic secant (HS) distribution if its probability density function (with unit variance) is given by

$$
f_{H S}(x)=\frac{1}{2 \cosh (\pi x / 2)}, \quad x \in \mathbb{R},
$$

or, equivalently, its cumulative distribution function is given by

$$
F_{H S}(x)=\frac{1}{2}+\frac{1}{\pi} \arctan (\sinh (\pi x / 2)) .
$$

This distribution is more leptokurtic than the normal, even more leptokurtic than the logistic distribution and has a kurtosis coefficient (measured by the fourth standardized moment) of 5. Consequently, it seems to be a reasonable "starting point" as a distribution for leptokurtic data, in particular for financial return data. In order to obtain higher "leptokurtic flexibility", the $\lambda$-th convolution of a hyperbolic secant distribution can be considered. 
This was discussed, for example, by Harkness and Harkness (1968) or Jørgensen (1997). The resulting distribution is commonly known as generalized hyperbolic secant (GHS) distribution. However, GHS offers still no opportunity to take skewness into account.

\subsection{NEF-GHS Distribution}

The NEF-GHS distribution was originally introduced by Morris (1982) in the context of natural exponential families (NEF) with specific quadratic variance functions. Densities of natural exponential families are of the form

$$
f(x ; \lambda, \theta)=\exp \{\theta x-\psi(\lambda, \theta)\} \zeta(x, \lambda) .
$$

In the case of the NEF-GHS distribution, $\psi(\lambda, \theta)=-\lambda \log (\cos (\theta))$ and $\zeta(x, \lambda)$ equals the probability density function of a generalized hyperbolic secant (GHS) distribution. Hence, the probability density function of the NEF-GHS distribution is given by

$$
f(x ; \lambda, \theta)=\underbrace{\frac{2^{\lambda-2}}{\pi \Gamma(\lambda)}\left|\Gamma\left(\frac{\lambda+i x}{2}\right)\right|^{2}}_{C(x)} \exp \{\theta x+\lambda \log (\cos (\theta))\}
$$

for $\lambda>0$ and $|\theta|<\pi / 2$. Introducing a scale parameter $\delta>0$ and a location parameter $\mu \in \mathbb{R}$, and setting $\beta \equiv \tan (\theta) \in \mathbb{R}$, equation (2) changes to

$$
f(x)=C\left(\frac{x-\mu}{\delta}\right) \exp \left(\arctan (\beta) \frac{x-\mu}{\delta}+\lambda \log (\cos (\arctan (\beta)))\right) .
$$

The NEF-GHS distribution is a flexible class of distributions which allows for skewness and excess kurtosis, which is infinitely divisible with existing moment-generating function and hence, existing moments. However, the cumulative distribution function and the inverse cumulative distribution function is not available in a closed form. These properties facilitate calculating risk measures, constructing multivariate copula-based distributions (see, e.g., Fischer, 2003).

\subsection{GSH distribution}

Another generalization of the hyperbolic secant distribution, which is able to model both thin and fat tails, was introduced by Vaughan (2002). This distribution family, the socalled standard generalized secant hyperbolic (GSH) distribution with kurtosis parameter $t \in(-\pi, \infty)$, has density

$$
f_{G S H}(x ; t)=c_{1}(t) \frac{\exp \left(c_{2}(t) x\right)}{\exp \left(2 c_{2}(t) x\right)+2 a(t) \exp \left(c_{2}(t) x\right)+1}, \quad x \in \mathbb{R}
$$

with

$$
\begin{array}{lll}
a(t)=\cos (t), & c_{2}(t)=\sqrt{\left(\pi^{2}-t^{2}\right) / 3}, & c_{1}(t)=c_{2}(t) \sin (t) / t, \quad \text { for }-\pi<t \leq 0 \\
a(t)=\cosh (t), & c_{2}(t)=\sqrt{\left(\pi^{2}+t^{2}\right) / 3}, & c_{1}(t)=c_{2}(t) \sinh (t) / t, \quad \text { for } t>0 .
\end{array}
$$


The density from (4) is chosen so that $X$ has zero mean and unit variance. The GSH distribution includes the logistic distribution $(t=0)$ and the hyperbolic secant distribution $(t=-\pi / 2)$ as special cases and the uniform distribution on $(-\sqrt{3}, \sqrt{3})$ as limiting case for $t \rightarrow \infty$. Vaughan (2002) derives the cumulative distribution function, depending on the parameter $t$, as

$$
F_{G S H}(x ; t)= \begin{cases}1+\frac{1}{t} \operatorname{arccot}\left(-\frac{\exp \left[c_{2}(t) x\right]+\cos (t)}{\sin (t)}\right) & \text { for } t \in(-\pi, 0), \\ \frac{\exp (\pi x / \sqrt{3})}{1+\exp (\pi x / \sqrt{3})} & \text { for } t=0, \\ 1-\frac{1}{t} \operatorname{arccoth}\left(\frac{\exp \left[c_{2}(t) x\right]+\cosh (t)}{\sinh (t)}\right) & \text { for } t>0,\end{cases}
$$

and the inverse cumulative distribution function

$$
F_{G S H}^{-1}(u ; t)= \begin{cases}\frac{1}{c_{2}(t)} \log \left(\frac{\sin (t u)}{\sin [t(1-u)]}\right) & \text { for } t \in(-\pi, 0) \\ \frac{\sqrt{3}}{\pi} \log \left(\frac{u}{1-u}\right) & \text { for } t=0 \\ \frac{1}{c_{2}(t)} \log \left(\frac{\sinh (t u)}{\sinh [t(1-u)]}\right) & \text { for } t>0\end{cases}
$$

The moment-generating function also depends on $t$ and is given by

$$
\mathcal{M}_{G S H}(u ; 0,1, t)= \begin{cases}\frac{\pi}{t} \sin \left(\frac{t u}{c_{2}(t)}\right) \csc \left(\frac{u \pi}{c_{2}(t)}\right) & \text { for } t \in(-\pi, 0), \\ \sqrt{3} u \csc (\sqrt{3} u) & \text { for } t=0, \\ \frac{\pi}{t} \sinh \left(\frac{t u}{c_{2}(t)}\right) \csc \left(u \pi / c_{2}(t)\right) & \text { for } t>0,\end{cases}
$$

where $\csc (\cdot)$ denotes the cosecans function. Moments of $X$ can be deduced from the last equation. Despite of its nice properties, the GSH distribution offers no possibility to include skewness effects.

\subsection{SGSH distributions}

There are plenty of methods in the literature to make a symmetric distribution skew. As the cumulative distribution function of the GSH distribution is explicitly known, Fischer and Vaughan (2002) decided in favour of splitting the scale parameter, as it was done by Fernández et al. (1995) for the skewed exponential power distribution.

Let $\gamma>1, \mathbf{I}^{+}(x)$ denote the indicator function for $x$ on $\mathbb{R}_{+}$and $\mathbf{I}^{-}(x)$ denote the indicator function for $x$ on $\mathbb{R}_{-}$. Then it can be easily verified that

$$
f_{S G S H}(x ; t, \gamma)=\frac{2}{\gamma+\frac{1}{\gamma}}\left\{f_{G S H}(x / \gamma) \cdot \mathbf{I}^{-}(x)+f_{G S H}(\gamma x) \cdot \mathbf{I}^{+}(x)\right\}
$$




$$
=\frac{2 c_{1}}{\gamma+\frac{1}{\gamma}} \cdot\left(\frac{\exp \left(c_{2} x / \gamma\right) \cdot \mathbf{I}^{-}(x)}{\exp \left(2 c_{2} x / \gamma\right)+2 a \exp \left(c_{2} x / \gamma\right)+1}+\frac{\exp \left(c_{2} \gamma x\right) \cdot \mathbf{I}^{+}(x)}{\exp \left(2 c_{2} \gamma x\right)+2 a \exp \left(c_{2} \gamma x\right)+1}\right)
$$

is a density function which is symmetric for $\gamma=1$, skewed to the right for $\gamma>1$ and skewed to the left for $0<\gamma<1$. The corresponding distribution is termed as skewed generalized secant hyperbolic distribution in the sequel. The effect of the skewness parameter on the density can be seen in Figure 1.

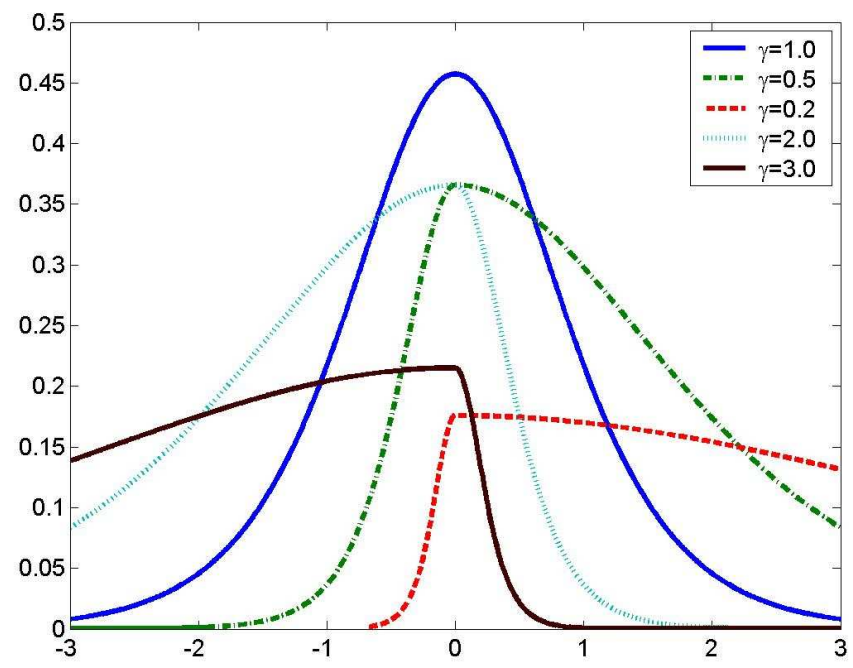

Figure 1: Effect of the skewness parameter.

It can also be shown (see, e.g., Grottke, 2001, p. 21) that the cumulative and the inverse cumulative distribution functions admits a closed form, namely

$$
\begin{aligned}
& F_{S G S H}(x ; t, \gamma)=\frac{2 \gamma^{2}}{\gamma^{2}+1}\left(F_{G S H}(x / \gamma) \mathbf{I}^{-}(x)+\left(\frac{\gamma^{2}-1+2 F_{G S H}(\gamma x)}{2 \gamma^{2}}\right) \mathbf{I}^{+}(x)\right), \\
& F_{S G S H}^{-1}(u ; t, \gamma)=\gamma F_{G S H}^{-1}\left(u \frac{\gamma^{2}+1}{2 \gamma^{2}}\right) \mathbf{I}^{A}(u)+\frac{1}{\gamma} F_{G S H}^{-1}\left(u \frac{\gamma^{2}+1}{2}-\frac{\gamma^{2}-1}{2}\right) \mathbf{I}^{\bar{A}}(u),
\end{aligned}
$$

with

$$
\mathbf{I}^{A}(u)=\left\{\begin{array}{l}
1, \text { if } u<\frac{2 \gamma^{2}}{1+\gamma^{2}} \\
0, \text { if } u \geq \frac{2 \gamma^{2}}{1+\gamma^{2}}
\end{array} \quad \text { and } \quad \mathbf{I}^{\bar{A}}(u)=1-\mathbf{I}^{A}(u) .\right.
$$

In addition, the power moments of a SGSH distribution can be deduced from that of a GHS distribution by means of

$$
\mathrm{E}\left(X_{S G S H}^{r}\right)=\mathrm{E}^{+}\left(X_{G S H}^{r}\right) \frac{2 \gamma}{\gamma^{2}+1}\left[(-1)^{r} \gamma^{r+1}+\gamma^{-r-1}\right]
$$

with the partial positive expectation value

$$
\mathrm{E}^{+}\left(X_{G S H}^{r}\right)=\int_{0}^{\infty} x^{r} f_{G S H}(x) d x
$$


Note, that for even $r$ the expectation of the positive part $\mathrm{E}^{+}\left(X_{G S H}^{r}\right)$ can be deduced from $\mathrm{E}\left(X_{G S H}^{r}\right)$ by division with 2 as

$$
\mathrm{E}\left(X_{G S H}^{r}\right)=2 \mathrm{E}^{+}\left(X_{G S H}^{r}\right)
$$

For $o d d r$, the formula for the half moments $\mathrm{E}^{+}\left(X_{G S H}^{r}\right)$ is slightly more complicated. The corresponding results are given by Fischer and Vaughan (2002).

\section{Financial Application of the SGSH distribution}

\subsection{The data set}

In order to adopt and compare estimation results for a great deal of distributions, in particular the stable distributions (STABLE), priority is given to the weekly returns of the Nikkei from July 31, 1983 to April 9, 1995, with $N=608$ observations. This series was intensively investigated, for example, by Mittnik et al. (1998) because it exhibits typical stylized facts of financial return data. Figure 2 illustrates the time series of levels and corresponding log-returns.

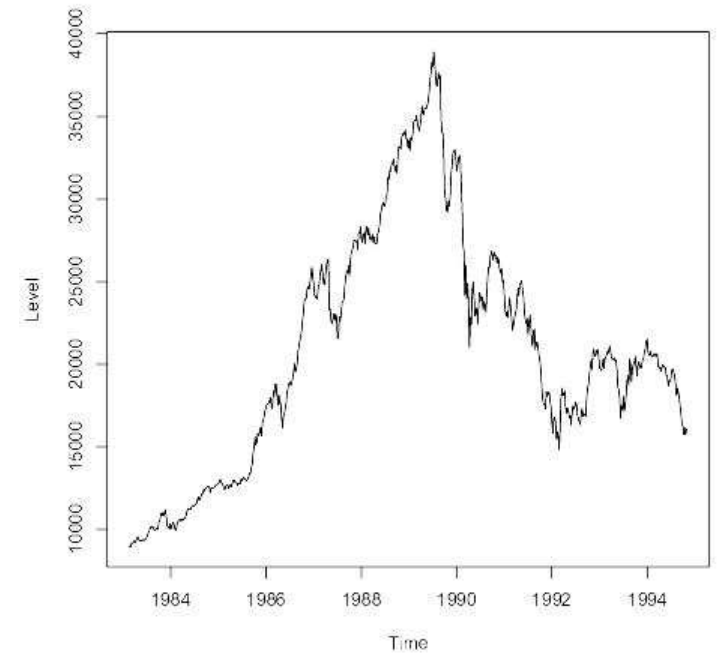

(a) Levels

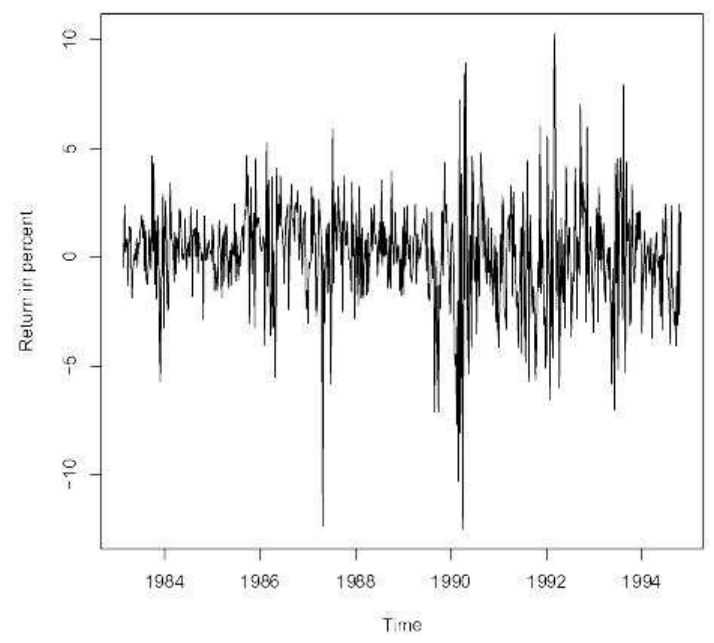

(b) Returns

Figure 2: Levels and returns of Nikkei.

\subsection{Unconditional fit to financial return data}

Similar to Mittnik et al. (1998), four criteria are employed to compare the goodnessof-fit of the different candidate distributions. The first is the log-Likelihood value $(\mathcal{L} \mathcal{L})$ obtained from the Maximum-Likelihood estimation. The $\mathcal{L} \mathcal{L}$-value can be considered as an "overall measure of goodness-of-fit and allows us to judge which candidate is more 
likely to have generated the data". As distributions with different numbers of parameters $k$ are used, this is taken into account by calculating the Akaike criterion given by

$$
A I C=-2 \mathcal{L} \mathcal{L}+\frac{2 N(k+1)}{N-k-2} .
$$

The third criterion is the Kolmogorov-Smirnov distance as a measure of the distance between the estimated parametric cumulative distribution function, $\hat{F}$, and the empirical sample distribution, $F_{e m p}$. It is usually defined by

$$
\mathcal{K}=100 \sup _{x \in \mathbb{R}}\left|F_{\text {emp }}(x)-\hat{F}(x)\right| .
$$

Finally, the Anderson-Darling statistic is calculated, which weights $\left|F_{\text {emp }}(x)-\hat{F}(x)\right|$ by the reciprocal of the standard deviation of $F_{e m p}$, namely $(\hat{F}(x)(1-\hat{F}(x)))^{1 / 2}$, that is

$$
\mathcal{A D}_{0}=\sup _{x \in \mathbb{R}} \frac{\left|F_{\text {emp }}(x)-\hat{F}(x)\right|}{\sqrt{\hat{F}(x)(1-\hat{F}(x))}} .
$$

Instead of just the maximum discrepancy, the second and third largest value, which are commonly termed as $\mathcal{A D}_{1}$ and $\mathcal{A D}_{2}$, are also taken into consideration. Whereas $\mathcal{K}$ emphasizes deviations around the median of the fitted distribution, $\mathcal{A D}_{0}, \mathcal{A D}_{1}$ and $\mathcal{A D}_{2}$ allow discrepancies in the tails of the distribution to be appropriately weighted.

Estimation was performed not only for the two families of generalized hyperbolic secant distributions (NEF-GHS and SGSH with symmetric counterparts GHS and GSH), but also for distribution families which have became popular in finance in the last years: Firstly, the generalized hyperbolic (GH) distributions which were discussed by Prause (1999) and include, for example, the Normal-inverse Gaussian (NIG) distributions (see Barndorff-Nielsen, 1977) as well as the hyperbolic (HYP) distributions (see Eberlein and Keller, 1995) as special cases. Secondly, the exponential generalized beta of the second kind (EGB2) distribution that was introduced by McDonald (1991) as a generalization of the logistic (LOG) distribution and used in various financial applications, see also Fischer (2002). Thirdly, a very flexible generalization of the generalized t-distribution (SGT2) proposed by Grottke (2001). Finally, we performed calculations for the gh-transformed normal (gh-NORM) distribution (see Klein and Fischer, 2002).

The estimation results are summarized in Table 1 and are as follows. Firstly, let us focus on the fit of generalized hyperbolic secant families. There seems to be no difference between the GSH distribution of Vaughan (2002) and the GHS distribution of Harkness and Harkness (1968). This is not true if we consider the skewed pendants and compare the NEF-GHS distribution of Morris (1982) with the SGSH distribution which exhibits better goodness-of-fit values with respect to all five criteria. For that reason, we restrict our considerations to the SGSH distribution. Concerning the $\mathcal{L} \mathcal{L}$-value, only the SGT2 distribution has a (slightly) higher value. The same is true if we compare the $\mathcal{K}$-values. If we take the number of parameters into account (i.e., focus on the $A I C$ criterion), SGSH even outperforms SGT2. The situation is a little bit different concerning the tail fit. Here, gh-transformed distributions finished best, followed by SGT2, NIG, STABLE and SGSH (Note, that the last three are close together). 
Table 1: Goodness-of-fit for the unconditional case: Nikkei225.

\begin{tabular}{lrrrrrrc} 
Distribution & $k$ & $\mathcal{L} \mathcal{L}$ & $\mathbf{A I C}$ & $\mathcal{K}$ & $\mathcal{A D}_{\mathbf{0}}$ & $\mathcal{A D}_{\mathbf{1}}$ & $\mathcal{A D}_{\mathbf{2}}$ \\
\hline NORM & 2 & -1428.3 & 2862.6 & 6.89 & 4.920 & 2.810 & 1.070 \\
STABLE & 4 & -1393.2 & 2796.5 & 3.00 & 0.085 & 0.084 & 0.081 \\
HS & 2 & -1393.4 & 2792.8 & 4.31 & 0.216 & 0.150 & 0.121 \\
GHS & 3 & -1392.2 & 2794.6 & 4.15 & 0.140 & 0.117 & 0.114 \\
NEF-GHS & 4 & -1388.1 & 2786.3 & 2.42 & 0.091 & 0.090 & 0.083 \\
GSH & 3 & -1392.3 & 2794.8 & 4.17 & 0.142 & 0.117 & 0.114 \\
SGSH & 4 & -1387.5 & $\mathbf{2 7 8 5 . 2}$ & 2.18 & 0.088 & 0.087 & 0.080 \\
LOG & 2 & -1398.1 & 2802.1 & 4.56 & 0.362 & 0.236 & 0.186 \\
EGB2 & 4 & -1388.1 & 2786.3 & 2.45 & 0.103 & 0.100 & 0.095 \\
GH & 5 & -1388.0 & 2788.2 & 2.43 & 0.095 & 0.093 & 0.086 \\
HYP & 4 & -1388.2 & 2786.5 & 2.50 & 0.106 & 0.103 & 0.098 \\
NIG & 4 & -1388.2 & 2786.6 & 2.48 & 0.085 & 0.085 & 0.075 \\
SGT2 & 5 & $\mathbf{- 1 3 8 7 . 4}$ & 2786.9 & $\mathbf{2 . 1 2}$ & 0.076 & 0.072 & 0.071 \\
Student-t & 3 & -1392.2 & 2792.5 & 3.77 & 0.107 & 0.104 & 0.103 \\
gh-NORM & 4 & -1388.7 & 2788.5 & 2.27 & $\mathbf{0 . 0 6 8}$ & $\mathbf{0 . 0 6 2}$ & $\mathbf{0 . 0 6 1}$
\end{tabular}

\subsection{Conditional fit to financial return data}

Assuming independent observations, as we did it in the last subsection, is not very realistic. To capture dependency between different log-returns, generalized autoregressive conditionally heteroscedastic (GARCH) models have proposed by Engle (1982) and Bollerslev (1986) as models for financial return data. These models are able to capture the distributional stylized facts (like thick tails or high peakedness), on the one hand, as well as the time series stylized facts (like volatility clustering), on the other hand. The setting for our GARCH framework is similar to Bollerslev (1986) assuming that the log-returns $R_{t}$ of financial data are given by

$$
\Theta_{m}(L) R_{t}=\mu+U_{t}
$$

with

$$
U_{t} \mid F_{t-1} \sim D\left(0, h_{t}^{2}, \eta\right) \quad \text { or } \quad U_{t} \mid F_{t-1}=h_{t} \epsilon_{t} \quad \text { with } \epsilon_{t} \sim D(0,1, \eta),
$$

where $\Theta_{m}(L)$ is a polynomial in the lag operator $L$ of order $m$. For reasons of simplicity, assume that $\Theta_{m}(L) \equiv 1$ and $\mu \equiv 0$. The residuals $\left\{U_{t}\right\}$ are assumed to follow a GARCHD process. That means they follow a distribution ${ }^{1} D$ with shape parameter $\eta$ and timevarying variance $h_{t}^{2}$. In the $\operatorname{GARCH}(1,1)$-Normal specification from Bollerslev (1986) it

\footnotetext{
${ }^{1}$ Although GARCH models with conditionally normally distributed errors imply unconditionally leptocurtic distributions, there is evidence (see, e.g., Bollerslev, 1987) that starting with leptocurtic and possibly skewed (conditional) distribution will achieve better results. For that reason, alternative error distributions are used.
} 
is given by

$$
h_{t}^{2}=\alpha_{0}+\alpha_{1} R_{t-1}^{2}+\beta_{1} h_{t-1}^{2}=\alpha_{0}+\alpha_{1} h_{t-1}^{2} \epsilon_{t-1}^{2}+\beta_{1} h_{t-1}^{2}
$$

Note, that setting $\beta_{1}=0$ results in the ARCH model of Engle (1982). The estimation results for the standard GARCH setting are summarized in Table 2.

Table 2: Goodness-of-fit for GARCH(1, 1)-models: Nikkei225.

\begin{tabular}{lrrrrrrc} 
Distribution & $k$ & $\mathcal{L} \mathcal{L}$ & $\mathbf{A I C}$ & $\mathcal{K}$ & $\mathcal{A D}_{\mathbf{0}}$ & $\mathcal{A D}_{\mathbf{1}}$ & $\mathcal{A D}_{\mathbf{2}}$ \\
\hline NORM & 4 & -1358.7 & 2727.8 & 4.31 & $>10$ & 0.890 & 0.141 \\
STABLE & 6 & -1340.8 & 2695.8 & 3.76 & 0.253 & 0.092 & 0.093 \\
HS & 4 & -1342.8 & 2695.6 & 4.54 & 0.427 & 0.126 & 0.125 \\
GHS & 5 & -1342.3 & 2698.7 & 4.01 & 0.679 & 0.122 & 0.122 \\
NEF-GHS & 6 & -1334.6 & 2683.4 & 2.25 & 0.461 & 0.083 & 0.076 \\
GSH & 5 & -1341.3 & 2696.8 & 3.71 & 0.914 & 0.155 & 0.116 \\
SGSH & 6 & 1333.0 & 2680.2 & $\mathbf{1 . 9 8}$ & 0.504 & 0.069 & 0.068 \\
LOG & 4 & -1344.0 & 2698.2 & 3.55 & 0.966 & 0.151 & 0.112 \\
EGB2 & 6 & -1335.6 & 2685.4 & 2.60 & 0.973 & 0.086 & 0.062 \\
GH & 7 & -1331.3 & 2678.8 & 2.27 & $\mathbf{0 . 1 5 0}$ & 0.062 & 0.061 \\
HYP & 6 & -1333.3 & 2680.9 & 2.21 & 0.834 & 0.079 & 0.063 \\
NIG & 6 & -1332.5 & 2679.1 & 2.19 & 0.466 & 0.065 & 0.064 \\
SGT2 & 7 & $\mathbf{- 1 3 3 1 . 1}$ & $\mathbf{2 6 7 8 . 4}$ & 2.32 & 0.172 & $\mathbf{0 . 0 5 7}$ & $\mathbf{0 . 0 5 6}$ \\
Student-t & 5 & -1340.3 & 2692.7 & 3.81 & 0.267 & 0.119 & 0.118
\end{tabular}

Again, the SGSH distribution outperforms most of its competitors and even has the lowest $\mathcal{K}$-value. Concerning the $\mathcal{L} \mathcal{L}$-value, only SGT2 and GH produce slightly higher values. The same is true for $\mathcal{A D}_{0}$.

GARCH models have been generalized in many different ways. In order to capture leverage effects, Zakoian (1994) proposed the threshold (T)-GARCH model with standard deviation given by

$$
h_{t}=\alpha_{0}+\alpha_{1}^{+} R_{t-1}^{+}-\alpha_{1}^{-} R_{t-1}^{-}+\beta_{1} h_{t-1},
$$

where $R_{t}^{+}=\max \left\{R_{t}, 0\right\}$ and $R_{t}^{-}=\min \left\{R_{t}, 0\right\}$. Imposing a Box-Cox-transformation on the conditional standard deviation process and the asymmetric absolute returns lead to the asymmetric power (AP-ARCH) specification of Ding et al. (1993), namely

$$
h_{t}^{\lambda}=\alpha_{0}+\alpha_{1}\left(\left|R_{t-1}\right|-c R_{t-1}\right)^{\lambda}+\beta_{1} h_{t-1}^{\lambda} .
$$

Equation (9) reduces to (8) for $\lambda=1, \alpha_{1}=\alpha_{1}^{-} /\left(2-\alpha_{1}^{+}\right)$and $c=1-\alpha_{1}^{+}\left(2-\alpha_{1}^{+}\right) / \alpha_{1}^{-}$. Moreover, equation (7) is achieved for $\lambda=2$ and $c=0$.

To take also asymmetric effects into account, we end up with an $\operatorname{AP}-\operatorname{ARCH}(1,1)-D$ specification where $D$ is chosen as in the $\operatorname{GARCH}(1,1)$ specification. Again, the results are summarized and given in Table 3 . Note that $\operatorname{AP}-\operatorname{ARCH}(1,1)$ estimation results for 
Table 3: Goodness-of-fit for AP-ARCH(1, 1)-models: Nikkei225.

\begin{tabular}{lrrrrrrr} 
Distribution & $k$ & $\mathcal{L} \mathcal{L}$ & $\mathbf{A I C}$ & $\mathcal{K}$ & $\mathcal{A D}_{\mathbf{0}}$ & $\mathcal{A D}_{\mathbf{1}}$ & $\mathcal{A D}_{\mathbf{2}}$ \\
\hline NORM & 6 & -1352.8 & 2719.8 & 4.28 & $>10$ & 0.473 & 0.125 \\
HS & 6 & -1339.7 & 2693.5 & 3.98 & 0.489 & 0.133 & 0.128 \\
GHS & 7 & -1338.6 & 2695.5 & 3.52 & 1.045 & 0.119 & 0.120 \\
NEF-GHS & 8 & -1333.7 & 2685.7 & 2.08 & 0.500 & 0.079 & 0.077 \\
GSH & 7 & -1337.3 & 2693.0 & 3.31 & 1.093 & 0.117 & 0.115 \\
SGSH & 8 & 1331.8 & 2681.9 & $\mathbf{1 . 8 9}$ & 0.543 & 0.073 & 0.067 \\
LOG & 6 & -1339.6 & 2693.4 & 3.08 & 1.183 & 0.113 & 0.112 \\
EGB2 & 8 & -1334.6 & 2687.5 & 2.46 & 1.011 & 0.078 & 0.077 \\
GH & 9 & $* *$ & $* *$ & $* *$ & $* *$ & $* *$ & $* *$ \\
HYP & 8 & -1332.5 & 2683.2 & 2.09 & 0.899 & 0.078 & 0.073 \\
NIG & 8 & -1331.6 & 2681.8 & 2.07 & 0.502 & 0.087 & 0.075 \\
SGT2 & 9 & $\mathbf{- 1 3 2 9 . 8}$ & $\mathbf{2 6 7 9 . 9}$ & 2.26 & $\mathbf{0 . 1 9 2}$ & $\mathbf{0 . 0 6 5}$ & $\mathbf{0 . 0 6 2}$ \\
Student-t & 7 & -1336.1 & 2688.3 & 3.28 & 0.398 & 0.108 & 0.105
\end{tabular}

stable distribution were not available. Moreover, $* *$ indicates problems with respect to convergence.

It can be observed that for most of the distributions the $\mathcal{K}$-values improve (compared to the GARCH(1,1)-fit) whereas the $\mathcal{A D}$-values become worse. SGT2 and SGSH seem to dominate the other distributions. Except of $\mathcal{A D}_{0}$, SGSH is close to SGT2. Again, SGSH achieves the smallest $\mathcal{K}$-value.

\section{Conclusions}

Two generalizations of the hyperbolic secant distribution have been proposed in the last years which seem to be encouraging as model for financial return data: The NEF-GHS distribution of Morris (1982) and the SGSH distribution of Fischer and Vaughan (2002). Both incorporate skewness and leptokurtosis. Within this work we applied them to the weekly log-returns of the Japanese stock index. Firstly, results of the unconditional fit were calculated as a benchmark. In a second step, volatility cluster are taken into account by means of a GARCH( 1,1$)$-model. Finally, we also tried to take care of leverage effects and estimated an $\operatorname{AP}-\operatorname{ARCH}(1,1)$-model. For the Nikkei data, we found that the skew generalized secant hyperbolic (SGSH) distribution provides an excellent fit in all cases. It dominates the NEF-GHS distribution as well as the EGB2 distribution. Furthermore, it approves as flexible but numerically easier to implement as the generalized hyperbolic family. Only SGT2, for which neither the cumulative distribution function nor the inverse cumulative distribution function are known, slightly outperforms SGSH. 


\section{References}

Barndorff-Nielsen, O. E. (1977). Normal-inverse Gaussian processes and stochastic volatility modelling. Scandinavian Journal of Statistics, 24, 1-13.

Baten, W. D. (1934). The probability law for the sum of $n$ independent variables, each subject to the law $(1 / 2 \mathrm{~h}) \operatorname{sech}(\pi \mathrm{x} / 2 \mathrm{~h})$. Bulletin of the American Mathematical Society, 40, 284-290.

Bollerslev, T. (1986). Generalized autoregressive conditional heteroskedasticity. Journal of Econometrics, 31, 302-327.

Bollerslev, T. (1987). A conditional heteroscedastic time series model for speculative price and rate of return. Review of Economics and Statistics, 9, 542-547.

Ding, Z., Engle, R. F., and Granger, C. W. J. (1993). A long memory property of stock markets returns and a new model. Journal of Empirical Finance, 1, 83-106.

Eberlein, E., and Keller, U. (1995). Hyperbolic distributions in finance. Bernoulli, 3, 281-299.

Engle, R. F. (1982). Autoregressive conditional heteroskedasticity with estimates of the variance of the United Kingdom inflation. Econometrica, 50, 987-1007.

Fernández, C., Osiewalski, J., and Steel, M. F. J. (1995). Modelling and inference with $\nu$ spherical distributions. Journal of American Statistical Association, 90, 1331-1340.

Fischer, M. (2002, June). The NEF-GHS option pricing model (Tech. Rep. No. 42). Universität Erlangen-Nürnberg: Lehrstuhl für Statistik und Ökonometrie.

Fischer, M. (2003, January). Tailoring copula-based multivariate skewed generalized secant hyperbolic distributions to financial return data: An empirical investigation (Tech. Rep. No. 47). Universität Erlangen-Nürnberg: Lehrstuhl für Statistik und Ökonometrie.

Fischer, M., and Vaughan, D. (2002, December). Classes of skewed generalized secant hyperbolic distributions (Tech. Rep. No. 45). Universität Erlangen-Nürnberg: Lehrstuhl für Statistik und Ökonometrie.

Grottke, M. (2001). Die t-Verteilung und ihre Verallgemeinerungen als Modell für Finanzmarktdaten. Lothmar: Josef Eul Verlag.

Harkness, W. L., and Harkness, M. L. (1968). Generalized hyperbolic secant distributions. Journal of the American Statistical Association, 63, 329-337.

Jørgensen, B. (1997). The theory of dispersion models. Chapman \& Hall.

Klein, I., and Fischer, M. (2002). Families of gh-transformed distributions. In S. Mittnik and I. Klein (Eds.), Contribution to modern econometrics. Kluwer.

McDonald, J. B. (1991). Parametric models for partial adaptive estimation with skewed and leptokurtic residuals. Econometric Letters, 37, 273-288.

Mittnik, S., Paolella, M. S., and Rachev, S. T. (1998). Unconditional and conditional distribution models for the Nikkei index. Asia-Pacific Financial Markets, 5, 99128.

Morris, C. N. (1982). Natural exponential families with quadratic variance functions. The Annals of Statistics, 10, 65-80.

Prause, K. (1999). The generalized hyperbolic model: Estimation, financial derivatives and risk measures. Unpublished doctoral dissertation, Universität Freiburg, Freiburg. 
Talacko, J. (1956). Perk's distributions and their role in the theory of Wiener's stochastic variables. Trabajos de Estadistica, 7, 159-174.

Vaughan, D. C. (2002). The generalized hyperbolic secant distribution and its application. Communications in Statistics - Theory and Methods, 31, 219-238.

Zakoian, J. M. (1994). Threshold heteroskedastic models. Journal of Economic Dynamics \& Control, 18, 931-955.

\author{
Author's address: \\ Dr. Matthias Fischer \\ Lehrstuhl für Statistik und Ökonometrie \\ Universität Erlangen-Nürnberg \\ Lange Gasse 20 \\ D-90403 Nürnberg \\ Tel. +609115320271 \\ Fax +60 9115320277 \\ E-mail: matthias.fischer@wiso.uni-erlangen.de \\ http://www.statistik.wiso.uni-erlangen.de
}

